\title{
LA FORMACIÓN EN INTERPRETACIÓN EN ESPAÑA: PASADO Y PRESENTE
}

\author{
Anne Martin \\ anne@ugr.es \\ Universidad de Granada
}

\section{Resumen}

Este artículo aborda la formación en interpretación en las universidades españolas desde que se inició en 1979. Se analizan los problemas asociados con el contenido en Interpretación de la Licenciatura en Traducción e Interpretación, vigente hasta hace poco, tales como la inclusión en el plan de estudios de asignaturas obligatorias que pretendían formar en las técnicas de interpretación utilizadas en Interpretación de Conferencia sin contar con la garantía de que los estudiantes cumplieran otros prerrequisitos necesarios para iniciar la formación en este género de interpretación. En la actualidad se está implantando la nueva titulación de Grado, y las universidades disfrutan de más libertad en la confección de sus planes de estudios. El panorama ahora es muy variado. Numerosas universidades han reducido sus créditos obligatorios en interpretación, al tiempo que han aumentado los créditos optativos. Aún así, sigue habiendo bastantes universidades que incluyen créditos obligatorios en interpretación simultánea. Parece que los títulos de Máster no acaban de consolidarse y otros géneros de interpretación (aparte de la interpretación de conferencia) han entrado en el plan de estudios.

\begin{abstract}
"Interpreter Training in Spain: Past and Present"

This paper deals with interpreter training in Spanish universities since its onset in 1979. Problems associated with the interpreting component in the recently phased out Licenciatura (4 year undergraduate course) in Translation and Interpreting are analysed. Such problems included the presence of compulsory subjects in the main conference interpreting techniques, without other prerequisites for beginning this training being guaranteed. At the current time a new degree is being introduced in the
\end{abstract}


framework of the EHEA (Grado, also a 4 year undergraduate course) which affords much more freedom to the universities in the design of their syllabus. The results are very varied. Many universities have reduced their compulsory credits in interpreting and now offer more optional credits. Despite this trend, there are still a significant number of universities with compulsory credits in simultaneous interpreting. Master's Degrees seem to be having difficulties in becoming consolidated and new genres of interpretation (other than conference interpreting) are being included in degree programmes.

Palabras clave: Formación en interpretación. Licenciatura en Traducción e Interpretación. Grado en Traducción e Interpretación. Interpretación simultánea. Interpretación de conferencia. Formación pregrado en interpretación.

Keywords: Interpreter training. Licenciatura in Translation and Interpreting. Grado in Translation and Interpreting. Simultaneous interpreting. Conference interpreting. Undergraduate training in interpreting.

Manuscript received on June 9, 2013 and accepted for publication on January 8, 2014. 


\section{Introducción}

Este artículo pretende abordar la formación de intérpretes en España, con especial referencia al nuevo panorama que se desarrolla en el marco del Espacio Europeo de Educación Superior. Para poder analizar el panorama actual, es necesario primero reflexionar sobre la evolución de la formación en interpretación en general y en España en particular. Con ese fin se propone una reflexión crítica sobre el componente de interpretación de la Licenciatura en Traducción e Interpretación y sus efectos. El objetivo último consiste en analizar el contenido de interpretación de las nuevas titulaciones implantadas en el marco del Espacio Europeo de Educación Superior (EEES) para identificar las tendencias de la formación en esta materia.

\section{Antecedentes de la formación en interpretación}

A diferencia de la formación de otros profesionales, la organización de la formación en interpretación como disciplina académica es relativamente reciente (al igual que ocurre con la Traducción), remontándose a mediados del siglo XX en algunos países de Europa, y a finales de la década de los años 70 en España. Esencialmente, se remonta a los años cincuenta con el auge de la interpretación de conferencia con posterioridad a la Segunda Guerra Mundial. Para entonces, la tecnología permitía la realización de interpretación simultánea, que se había utilizado por primera vez con éxito durante los juicios de Núremberg en 1945. Poco después, esta nueva modalidad de interpretación hizo posible la utilización de seis idiomas oficiales en la recién creada Organización de las Naciones Unidas (ONU). Sin embargo, la interpretación simultánea requería una formación sistematizada, y pronto aparecieron los primeros cursos de formación en interpretación de conferencia en algunas universidades alemanas (Heidelberg, Gemersheim), en la Universidad de Ginebra y la Sorbona de París.

Por otra parte, en esta época (1953) se creó AIIC (Association Internationale des Interprètes de Conférence) y se pusieron en marcha las instituciones europeas, con el consiguiente aumento de la demanda de intérpretes de conferencia. En este contexto del nacimiento de esta nueva profesión, se forjaron 
estrechos vínculos entre AIIC, las primeras escuelas de formación y las instituciones europeas. De hecho, en sus inicios, la formación de intérpretes de conferencia en Europa estuvo estrechamente vinculada con las necesidades de las organizaciones internacionales, y apoyada por AIIC. Durante este periodo inicial se consolidaron muchos aspectos de la profesión de intérprete de conferencia tal y como la conocemos hoy en día.

Fue entonces cuando nació la teoría del sentido o teoría interpretativa de la traducción, asociada principalmente con Danica Seleskovitch y Marianne Lederer de la ESIT, formadoras pioneras de la época e intérpretes profesionales. Dicha teoría está plasmada en las obras emblemáticas de Danica Seleskovitch Les Interprètes dans les Conférences Internationales (1968) y Langage, Langues et Mémoire (1975), a las que siguieron muchas otras publicaciones emanadas de la misma escuela. La obra más relevante sobre formación es Pédagogie Raisonnée de l'Interprétation (Seleskovitch y Lederer, 1989, 2002) que, indudablemente, marcó tendencia en la formación de intérpretes de conferencia en el mundo occidental.

No es este el lugar para emprender un análisis de la teoría del sentido, pero basta decir que significó un novedoso rechazo a las rígidas teorías de equivalencias lingüísticas y estructuralismo que caracterizaban algunos enfoques teóricos de la traducción en aquella época. Por otra parte, sirvió para distanciar claramente la formación en interpretación de la enseñanza de idiomas y también de la formación en traducción escrita.

En el tiempo transcurrido desde entonces, la teoría del sentido ha ido evolucionando, adaptándose y templándose con el paso del tiempo y el desarrollo de la profesión. Por otra parte, también han surgido otros planteamientos para enriquecer lo anterior y cuestionar algunos de sus preceptos. Nos referimos concretamente al modelo de esfuerzos de Gile (1985, 1991, 1995), la investigación en neurociencias llevada a cabo principalmente en la Universidad de Trieste (SSLMIT) (Gran 1989; Fabbro, Gran, Basso y Bava 1990; Fabbro y Gran 1994), y la investigación en psicología cognitiva (Lambert 1989; Padilla 1996) así como el trabajo de Pöchhacker (1995) al aplicar la teoría funcionalista a la interpretación.

Por tanto, en nuestros días, a diferencia de los años 70, la teoría del sentido ya no es el único modelo que busca explicar el proceso de interpretación de conferencia y servir de base para su pedagogía, sino que coexiste con otros modelos que se han elaborado desde entonces y que forman parte del fructífero campo de lo que ahora se llama Estudios de Interpretación.

Sin embargo, es indudable que la teoría del sentido, más que cualquier otro modelo teórico, ejerció y sigue ejerciendo una clara influencia en la 
pedagogía de la interpretación en general, tanto como explicación del proceso en sí cuanto como base de su pedagogía. Esta influencia se percibe en muchos países de Europa en la tendencia a favorecer la enseñanza de la interpretación de conferencia a nivel de posgrado previa realización de una prueba de aptitud para determinar que los candidatos a la formación reúnen las condiciones mínimas para iniciarla. Igualmente, se refleja en la idea de anteponer el aprendizaje de la consecutiva al de la simultánea, para fomentar la disociación entre palabra e idea, así como en la tendencia a evitar la simultánea inversa, por suponer, según la teoría del sentido, una merma de la calidad de la versión final, aunque este último precepto, que ha sido el sistema imperante en las instituciones europeas hasta hace poco, se ha visto alterado por razones prácticas con las sucesivas ampliaciones de la UE (Martin 2003).

Por otra parte, la vigencia actual del modelo de Seleskovitch y su influencia se percibe en varias prácticas de frecuente aplicación en nuestras aulas, por ejemplo la disociación de palabra y significado, así como la realización de ejercicios de pre-interpretación (análisis, memorización, ejercicios de agilidad y de oratoria pública) para la adquisición de destrezas de forma discreta, todas prácticas recomendadas por autores más recientes (Nolan 2005; Gillies 2004, 2013).

Estos fundamentos pedagógicos de la interpretación parecen suscitar un relativo consenso en Europa, y se aprecian claramente en las recomendaciones de AIIC sobre buenas prácticas en la formación de intérpretes (AIIC: en línea).

Estas prácticas reflejan, a grandes rasgos, la manera más difundida y eficaz de formar a intérpretes de conferencia en Europa en la actualidad: mediante una especialización de posgrado (o similar) para candidatos que han demostrado reunir las condiciones de partida para dicha formación (Kelly \& Martin 2009). La filosofía subyacente es la siguiente: si el estudiante posee las competencias instrumentales necesarias (excelente dominio de las lenguas de trabajo, conocimiento de la cultura y civilización de los países donde se hablan dichos idiomas y conocimiento de relaciones internacionales, política y economía), la competencia en interpretación (al igual que la de traducción) se puede adquirir en relativamente poco tiempo.

En este sentido, existe una iniciativa paneuropea de formación en interpretación de conferencia denominada European Master in Conference Interpreting (EMCI: en línea), impulsada por las instituciones europeas e impartida por un consorcio de universidades en los distintos Estados miembros de la UE en colaboración con la Comisión Europea y el Parlamento Europeo. En un principio, esta titulación obedecía a la necesidad de las instituciones europeas de 
contar con intérpretes profesionales en los idiomas de los países de las sucesivas ampliaciones de la UE, lenguas que no se solían enseñar en las escuelas y universidades de los países más veteranos. Sin embargo, también ha servido como vehículo para la formación de nuevas generaciones de intérpretes en las combinaciones lingüísticas más generales. En España, esta titulación se ofertó en la Universidad de La Laguna (ULL), en Canarias, desde 1988 hasta 2013 en un posgrado de tipo máster (título propio) de 60 créditos, de los cuales el $80 \%$ son créditos prácticos de interpretación de conferencia. ${ }^{1}$ Esto refleja la recomendación por parte del consorcio de dedicar un mínimo de 400 horas presenciales a la práctica de la interpretación para poder llegar a un nivel adecuado de práctica profesional.

\section{Formación en interpretación en la Universidad española}

\subsection{Diplomatura en Traducción e Interpretación}

La formación de intérpretes en la Universidad española se inició más tarde que en otros países europeos. Empezó como parte de la Diplomatura en Traducción e Interpretación de tres años de duración que se implantó en la Universidad Autónoma de Barcelona (UAB) en 1979, un año más tarde en la Universidad de Granada (UGR) y, en 1988, en la Universidad de Las Palmas de Gran Canaria (ULPGC). Durante este periodo se empezó a impartir un posgrado en interpretación de conferencia en la UAB, y en 1988 se inició el Máster en Interpretación de Conferencia de la ULL, como ya se ha señalado.

La Diplomatura dio paso a una Licenciatura en Traducción e Interpretación aprobada en 1991, fecha a partir de la cual se implanta la titulación en un número creciente de universidades por toda la geografía española hasta superar la veintena de centros, tanto públicos como privados, que hoy en día imparten enseñanzas en Traducción e Interpretación (Kelly 1996).

\subsection{Licenciatura en Traducción e Interpretación}

En las Directrices Generales de la Licenciatura, se incluía un total de 18 créditos troncales (y por tanto obligatorios) en interpretación, divididos en dos asignaturas: Técnicas de Interpretación Consecutiva y Técnicas de Interpretación Simultánea. Estas asignaturas estaban presentes en todas las universidades donde se impartía la titulación (Ministerio de Educación y Ciencia 1991).

1. La ULL sigue ofertando el Máster en Interpretación de Conferencias (MIC), si bien, formalmente, no forma parte del consorcio EMCI, que ha sufrido una profunda reorganización en los últimos años. 
Por tanto, coincidían los nombres de las asignaturas troncales con las dos técnicas de interpretación más ampliamente utilizadas en la interpretación de conferencia, estableciéndose desde el principio una identificación clara de la formación en la Licenciatura con este género de interpretación. El nombre asignado a estas asignaturas implica que el objetivo de la titulación era la formación de intérpretes de conferencia, aunque dicho objetivo no figuraba por escrito en los documentos oficiales de la titulación. Ya hemos visto que existe cierto consenso en torno a la tendencia del resto de Europa a formar intérpretes en posgrado o como especialización a finales de la carrera de pregrado, previa adquisición de las competencias instrumentales necesarias, y que este modelo está avalado por los principales empleadores de intérpretes, es decir, por las instituciones europeas y otros organismos internacionales. El consorcio europeo EMCI (en línea) recomienda que la formación en interpretación de conferencia conste de por lo menos 400 horas de clases prácticas presenciales impartidas por profesores que son intérpretes profesionales.

España, por tanto, empezó una formación en interpretación de conferencia que iba en contra de la experiencia acumulada de otros países. Por esta razón, consideramos que la Licenciatura nació potencialmente viciada de raíz en cuanto a su componente troncal de interpretación. Quizás uno de los principales errores del plan de estudios de la Licenciatura fue el considerar la interpretación de conferencia como el único género de interpretación existente, lo cual llevó a la inclusión de las técnicas de interpretación más usadas en ese género. Sin embargo, existen otros géneros de interpretación, tales como la interpretación de acompañamiento, la interpretación social/en servicios públicos, la interpretación para el comercio y el turismo, que no están asociados a las técnicas de consecutiva y simultánea, y sí a la técnica de interpretación de enlace, por ejemplo, más asequible para los estudiantes, máxime teniendo en cuenta el número limitado de horas y las carencias en competencias instrumentales de muchos estudiantes de pregrado. A pesar de estos condicionantes, estos otros géneros de interpretación no se tomaron en cuenta (Mayoral Asensio 2007, Calvo Encinas 2009).

Es cierto que las asignaturas troncales de Interpretación Consecutiva y Simultánea de la Licenciatura no necesariamente se enfocaban de la misma manera en todas las universidades donde se impartía la titulación. En algunas universidades se siguió el nombre de las asignaturas al pie de letra, procurando ofertar una formación que incluía la interpretación simultánea propiamente dicha. En otras, sin embargo, se procuró ofertar a los estudiantes una introducción a la Interpretación en general, obviando aquellas técnicas que resultan difíciles de adquirir con un número de créditos tan reducido y, 
en todo caso, centrándose en el desarrollo de las aptitudes necesarias para la posterior adquisición de la simultánea: fomentar la agilidad, la rapidez de reflejos y la atención dual, pero sin llegar a realizar interpretación simultánea propiamente dicha.

En algunas universidades se ofertaban asignaturas optativas adicionales para fomentar una mayor especialización en Interpretación. Concretamente, en la UGR se habilitó un itinerario optativo de último curso de especialización en Interpretación de Conferencia que constaba de 400 horas.

\subsection{Grado en Traducción e Interpretación}

En la actualidad se está completando la implantación de los nuevos títulos de Grado en Traducción e Interpretación en el marco del EEES. De acuerdo con la filosofía plasmada en los Descriptores de Dublín (VV.AA.), las competencias a las que llega un estudiante al finalizar sus estudios de Grado son generalistas, reservándose las competencias especializadas para la adquisición posterior (Calvo Encinas 2009).

En este nuevo panorama, ya no existen estipulaciones universales sobre el número de créditos, las denominaciones de las asignaturas, el programa de estudios y ni siquiera en cuanto al nombre de la titulación. Cada universidad tiene libertad para elaborar su propio plan de estudios, por lo que la situación es bastante dispar en relación con la Licenciatura. La salvedad a esta situación la constituye la Comunidad Autónoma andaluza, ya que la Junta de Andalucía obliga a las universidades de esta comunidad a consensuar el $75 \%$ del contenido en grados del mismo nombre. Esto significa que el $75 \%$ del contenido de los Grados en Traducción e Interpretación de las universidades andaluzas es común y los planes de estudio solo difieren el uno del otro en un $25 \%$ de su contenido.

A continuación, pasaremos a analizar el número de créditos en interpretación en las diferentes universidades donde se imparte la titulación de Grado en Traducción e Interpretación, análisis que abarca su contenido, su distribución y su lugar en el plan de estudios. ${ }^{2}$ El objetivo de este análisis es buscar posibles tendencias en cuanto a la formación en interpretación, ahora que cada universidad, con la excepción de las andaluzas, tiene libertad para

2. La denominación de la titulación de Grado con contenido en traducción no es uniforme en todas las universidades. Aunque en la mayoría se denomina Grado en Traducción e Interpretación, en la Universidad Europea de Madrid (UEM) se denomina Grado en Traducción y Comunicación Intercultural y en la Universidad de Alcalá (UAH) se denomina Grado en Lenguas Modernas y Traducción. Este último no oferta créditos de interpretación. 
configurar su propio plan de estudios. Los datos que aparecen a continuación se refieren a un total de veintidós universidades y han sido extraídos de tres fuentes principales: informe elaborado por el Grupo Comunica (en línea) con vistas a la elaboración del Libro Blanco sobre Traducción e Interpretación Institucional en España (RITAP y APTIJ 2011), páginas web de las universidades implicadas en el momento de escribir este capítulo y contactos personales en dichas universidades. Muchas de estas titulaciones están todavía en fase de implantación y algunas de las asignaturas no han sido activadas aún, por lo que cabe imaginar que podría haber algunas variaciones. Hemos considerado como "asignatura de interpretación" cualquier asignatura cuyo contenido incluye ejercicios de interpretación (consecutiva, simultánea, enlace, traducción a vista) o preinterpretación (ejercicios de expresión oral, análisis y síntesis, memorización, toma de apuntes, clozing, anticipación, etc.).

\subsubsection{Créditos obligatorios de interpretación}

En siete de las universidades que ofertan la titulación de Grado en Traducción e Interpretación el número de créditos obligatorios en interpretación es igual o superior a los 18 créditos obligatorios de la Licenciatura, como se puede apreciar en la Tabla 1.

TABLA 1. UNIVERSIDADES CON 18 O MÁS CRÉDITOS OBLIGATORIOS EN INTERPRETACIÓN

\begin{tabular}{|c|c|c|}
\hline UNIVERSIDAD & $\begin{array}{c}N^{o} \text { TOTAL de } \\
\text { CRÉDITOS }\end{array}$ & $\begin{array}{c}N^{o} \text { CRÉDITOS } \\
\text { SIM (INCLUIDOS } \\
\text { EN TOTAL) }\end{array}$ \\
\hline ALFONSO X EL SABIO (UAX) & 25 & 6 \\
\hline ALICANTE (UA) & 18 & 6 \\
\hline CÓRDOBA (UCO) & 18 & 0 \\
\hline $\begin{array}{l}\text { LAS PALMAS DE GRAN CANARIA } \\
\text { (ULPGC) }\end{array}$ & 30 & 12 \\
\hline MURCIA (UMU) & 18 & 6 \\
\hline PONTIFICIA DE COMILLAS (UPCO) & 24 & 0 \\
\hline VIGO (UVi) & 18 & 6 \\
\hline
\end{tabular}

Universidades como la UAX, la ULPGC y la UPCO ofertan un alto número de créditos obligatorios en interpretación en el marco del Grado y en algunas de estas universidades parte de estos créditos obligatorios se destinan a la enseñanza de la interpretación simultánea. Es el caso de la UAX, donde 6 de 
sus 25 créditos obligatorios son de simultánea; La ULPGC, donde 12 de los 30 se dedican a la simultánea; y UA, UVi y UMU con 6 de sus 18 créditos de simultánea.

Por el contrario, la UPCO, si bien oferta un alto número de créditos obligatorios en interpretación, no incluye la formación en interpretación simultánea entre dichos créditos. Ocurre lo mismo en la UCO. En la mayoría de los casos, las asignaturas obligatorias solo se refieren a la lengua B del estudiante, aunque en la UAX, UCO y la ULPGC también hay créditos obligatorios de interpretación en lengua $C$.

En el otro extremo (véase Tabla 2) existen universidades que ofertan un número mínimo de créditos obligatorios de interpretación y ningún crédito obligatorio en interpretación simultánea. Entre estas se encuentran la Universitat Autònoma de Barcelona (UAB), la Universidad Autónoma de Madrid (UAM), la Universidad del País Vasco (UPV), la Universidad San Jorge (USJ) y la Universidad Complutense de Madrid -CES Felipe II- (UCMF2) con 6 créditos obligatorios de Introducción a la Interpretación cada una, la Universitat Jaume I (UJI) y la Universidad Pompeu Fabra (UPF) con 8. Ninguno de estos créditos obligatorios se dedica a la enseñanza de la simultánea. En todos estos casos, con la excepción de la USJ, existe un itinerario optativo en interpretación. Estos itinerarios no se limitan a la interpretación de conferencia, aunque sigue siendo la oferta mayoritaria, y en algunos casos se trata de itinerarios muy completos, como el de 60 créditos ofertado por la UCM-F2. Por tanto, existe la posibilidad de especializarse en Interpretación en estos centros, de forma opcional, si así lo desea el estudiante.

\section{TABLA 2. UNIVERSIDADES CON MENOS CRÉDITOS OBLIGATORIOS EN INTERPRETACIÓN}

\begin{tabular}{|l|c|}
\hline \multicolumn{1}{|c|}{ UNIVERSIDAD } & $N^{\circ}$ CRÉDITOS \\
\hline UAB & 6 \\
\hline UAM & 6 \\
\hline UCM-F2 & 6 \\
\hline UJI & 8 \\
\hline UPV & 6 \\
\hline UPF & 8 \\
\hline USJ & 6 \\
\hline
\end{tabular}


En un término medio (véase Tabla 3) nos encontramos con otra serie de universidades como la UGR, Salamanca (USAL), Valencia (UVa), Málaga (UMA), la Europea de Madrid (UEM), la Universidad Pablo de Olavide (UPO), Valladolid (UV-Soria) y Vic (UVIC) que ofertan 12 créditos obligatorios en Interpretación cada una. Entre ellas, USAL, UV-Soria, UVIC y la UEM dedican parte de esos créditos a la enseñanza obligatoria de la simultánea. En el caso de Salamanca se trata de una "introducción a la simultánea", y en el caso de la UEM y las UV-Soria y UVIC la asignatura recibe la denominación de "Interpretación Simultánea".

TABLA 3. UNIVERSIDADES CON SITUACIÓN INTERMEDIA EN CUANTO A CRÉDITOS OBLIGATORIOS DE INTERPRETACIÓN

\begin{tabular}{|l|c|c|}
\hline \multicolumn{1}{|c|}{ UNIVERSIDAD } & $\begin{array}{c}\mathrm{N}^{o} \text { TOTAL DE } \\
\text { CRÉDITOS }\end{array}$ & $\begin{array}{c}\mathrm{N}^{o} \text { CRÉDITOS SIM } \\
\text { (INCLUIDOS EN } \\
\text { TOTAL) }\end{array}$ \\
\hline UEM & 12 & 6 \\
\hline UGR & 12 & 0 \\
\hline UMA & 12 & 0 \\
\hline UPO & 12 & 0 \\
\hline USAL & 12 & 6 \\
\hline UVa & 12 & 0 \\
\hline UV-Soria & 12 & 6 \\
\hline UVIC & 12 & 6 \\
\hline
\end{tabular}

De las veintidós universidades estudiadas hay nueve con créditos obligatorios de interpretación simultánea en el grado, que se pueden ver en la Tabla 4. El número de créditos en todos los casos es de 6, menos en la ULPGC donde suman 12. En la UMU, de acuerdo con los datos recogidos en su página web, los créditos obligatorios incluyen simultánea inversa, al igual que la Universidad de Alicante. 


\section{TABLA 4. UNIVERSIDADES CON CRÉDITOS OBLIGATORIOS DE INTERPRETACIÓN SIMULTÁNEA}

\begin{tabular}{|l|c|}
\hline & $\begin{array}{c}N^{o} \text { CRÉDITOS } \\
\text { OBLIGATORIOS EN INT } \\
\text { SIM }\end{array}$ \\
\hline UAX & 6 \\
\hline UA & 6 \\
\hline UEM & 6 \\
\hline ULPGC & 12 \\
\hline UMU & 6 \\
\hline USAL & 6 \\
\hline UV-Soria & 6 \\
\hline UVIC & 6 \\
\hline UVi & 6 \\
\hline
\end{tabular}

\subsubsection{Denominación de las asignaturas obligatorias}

Teniendo en cuenta que la formación en Interpretación en la Licenciatura se encontraba estrechamente condicionada por la denominación de las asignaturas, parece interesante comprobar si las universidades han aprovechado la oportunidad del nuevo plan de estudios para cambiar estos nombres o si han seguido con las antiguas denominaciones, referidas a técnicas específicas utilizadas en interpretación de conferencia.

En nueve de las universidades estudiadas, la titulación de Grado incluye asignaturas obligatorias con nombres referidos a la interpretación consecutiva y/o simultánea, similares a los de las asignaturas troncales de interpretación en la Licenciatura. Estas universidades son: UAX, ULPGC, UEM, UV-Soria, UMA, UMU, USAL, UVIC, UVi, lista que, a grandes rasgos, coincide con las universidades que aparecen en la Tabla $4 .{ }^{3}$

En las demás universidades las asignaturas obligatorias de interpretación tienen denominaciones muy generales que incluyen palabras como "Iniciación" (UAB, UJI), "Introducción" (UAM, UCM-F2) o "Fundamentos" (USAL). La UPF recalca la naturaleza no específica de sus asignaturas obligatorias al

3. Esta lista no coincide exactamente con la lista de universidades que tienen interpretación simultánea obligatoria, ya que en la UA el nombre de la asignatura es genérico, por lo que no aparece en esta lista y, en la UMA, la asignatura en cuestión es Interpretación Consecutiva (pero no Simultánea), por lo que sí aparece aquí, pero no entre las universidades que incluyen créditos obligatorios en simultánea. 
llamarlas "Técnicas de expresión oral". Solo la UCO oferta una asignatura aparentemente especializada "Interpretación Jurídica y Económica" entre sus asignaturas obligatorias.

Por tanto, en el panorama actual, por un lado hay universidades que otorgan el título de Grado en Traducción e Interpretación con un mínimo de créditos en Interpretación y con un contenido bastante general e introductorio: la UJI y la UPF con 8 créditos, la UAB, la UAM, la UPV y la USJ con 6. Por otro lado, hay universidades tales como la UAX y la ULPGC donde los estudiantes deben cursar un número importante de créditos obligatorios en interpretación, incluyendo la interpretación simultánea.

\subsubsection{Asignaturas optativas de interpretación}

Además de los créditos obligatorios en interpretación que deben existir a la fuerza para justificar el nombre de la titulación, también existe una amplia gama de asignaturas optativas en interpretación en la mayoría de las universidades estudiadas. Estos créditos optativos abarcan distintos géneros de interpretación y no solo se refieren a la interpretación de conferencia. Las excepciones son UCO, UVa, UA, y USJ, en las que hay menos de 6 créditos de optativas de interpretación en el Grado.

\subsubsection{Itinerarios específicos de interpretación}

Hay itinerarios específicos en interpretación, ofertados de forma optativa, en siete universidades, que son precisamente aquellas que tienen el menor nivel de obligatoriedad en interpretación (véase Tabla 5). En la UAB por ejemplo, donde hay 6 créditos obligatorios, se oferta un itinerario optativo de 30 créditos en interpretación; en la UAM donde, igualmente, hay 6 créditos obligatorios, se ofertan además unos 30 créditos optativos por lengua en un itinerario específico. En la UVIC, con 12 créditos obligatorios, hay un itinerario optativo con 24 créditos, y en la UCM-F2 los 6 créditos obligatorios se ven complementados por un itinerario específico de 60 créditos, como ya se señaló arriba. Este último itinerario incluye asignaturas en interpretación en los Servicios públicos. En las otras tres universidades con itinerario específico en interpretación (UMA, USAL y UJI) el número de créditos de optativas y obligatorias es similar, aunque el balance siempre se inclina a favor de los créditos optativos. En la UMA se ofertan 12 créditos obligatorios y 18 optativos; en la USAL 12 obligatorios y 15 optativos y en la UJI, 8 obligatorios y 13,5 optativos. 
TABLA 5. ITINERARIOS OPTATIVOS EN INTERPRETACIÓN

\begin{tabular}{|l|c|c|}
\hline \multicolumn{1}{|c|}{ UNIVERSIDAD } & $\begin{array}{c}\text { CRÉDITOS } \\
\text { OBLIGATORIOS }\end{array}$ & $\begin{array}{c}\text { CRÉDITOS } \\
\text { ITINERARIO } \\
\text { OPTATIVO }\end{array}$ \\
\hline UAB & 6 & 30 \\
\hline UAM & 6 & 30 \\
\hline UCM-F2 & 6 & 60 \\
\hline UJI & 8 & 13,5 \\
\hline UMA & 12 & 18 \\
\hline UPV & 6 & 24 \\
\hline UPF & 8 & 16 \\
\hline USAL & 12 & 15 \\
\hline UVIC & 12 & 24 \\
\hline
\end{tabular}

Hay otras universidades que, aunque no utilizan el término "itinerario" como tal, tienen pocos créditos obligatorios y mucha optatividad en interpretación. Un ejemplo sería la UPV con 6 créditos obligatorios y 24 optativos por combinación lingüística o la UPF, que si bien no habla de itinerario, sí usa el término "perfil" y oferta 8 créditos obligatorios y 16 optativos. En la UV-Soria tampoco se menciona la palabra "itinerario" pero existe un bloque diferenciado de asignaturas centradas, en este caso, en la interpretación social.

Se comentaba arriba que el plan de estudios de la Licenciatura, a través de las asignaturas troncales de interpretación, parecía centrarse en la interpretación de conferencia en detrimento de otros géneros que podrían ser de mayor utilidad para los estudiantes. En el Grado, algunas universidades parecen haber empezado a remediar esta situación.

En la nueva titulación se ofertan asignaturas optativas en otros géneros de interpretación que no sean la interpretación de conferencia en por lo menos 9 de las 22 universidades estudiadas. Los ámbitos abarcados son interpretación para entornos empresariales (UAX, UMU), interpretación para el comercio y turismo (UGR) e interpretación en los servicios públicos (UGR, UJI, UMU, UPV, UEM, UAX, UCM-F2 y USJ). Es interesante señalar que en la UPO, la UV-Soria y la UPF se oferta la posibilidad de hacer interpretación de lengua de signos, la cual abre las puertas a otras salidas laborales relacionadas con la interpretación en los servicios públicos.

Está claro que, después de la interpretación de conferencia, el campo más ampliamente abarcado es la interpretación en los servicios públicos (ISP) y, 
en cierta manera, no es de extrañar, teniendo en cuenta que este tipo de interpretación está en auge en España. Sin embargo, por otro lado, no deja de ser curioso que existan tal cantidad de iniciativas formativas universitarias en ISP, teniendo en cuenta que no existe un perfil profesional claro para esta actividad en España, donde está en una fase pre-profesional, y que aun no es una actividad profesional plenamente reconocida ni remunerada (Ortega Herráez, Abril Martí y Martin 2009). ${ }^{4}$

En suma, este breve estudio revela un panorama bastante dispar en cuanto al contenido en interpretación de los nuevos grados. Hay una serie de universidades con planes de estudio cuyo contenido en interpretación obligatoria es reducido e introductorio, pero que ofertan itinerarios con bastantes créditos optativos para posibilitar que quien quiera hacerlo pueda especializarse en interpretación. Por otra parte, hay un número significativo de universidades cuyo plan de estudios de Grado incluye muchos créditos obligatorios de interpretación, incluida interpretación simultánea.

\subsection{Posgrado en interpretación de conferencia}

Pasemos ahora a la formación en interpretación en el marco de los títulos de posgrado. Ya se ha mencionado que esta es la forma más recomendada a nivel europeo y, por otra parte, en términos generales, la filosofía del Espacio Europeo de Educación Superior descansa en los títulos de máster como vía de especialización del estudiante en todos los campos.

El posgrado de mayor tradición en interpretación de conferencia en España es el ya mencionado Máster en Interpretación de Conferencia de la ULL, puesto en marcha por las instituciones europeas e impartido ininterrumpidamente desde el año 1988 como miembro del consorcio EMCI. ${ }^{5}$ No se trata de un máster universitario oficial sino de un título propio de la universidad donde se imparte. La formación ofertada en esta titulación está ajustada a las necesidades de las instituciones europeas. Por otra parte, la UGR oferta el título oficial de Máster Universitario en Interpretación de Conferencia desde el curso 2013-14. Dicha titulación sigue las recomendaciones del consorcio EMCI y procura formar a los estudiantes tanto para el mercado institucional como el freelance.

Aparte de estas titulaciones, existen otras universidades que ofertan (según demanda) o han ofertado títulos de Máster en Interpretación de Conferencia

4. En España es muy frecuente que esta actividad la realicen voluntarios o miembros de la familia o del círculo más cercano al usuario

5. Esta titulación dejó de formar parte de la EMCI en fechas recientes. 
en el pasado, tales como la UVIC y la UPCO, o que tienen la titulación aprobada aunque no se ha impartido hasta la fecha, como es el caso de la UVi.

Por otra parte, hay titulaciones de posgrado que, si bien no se dedican exclusivamente a la formación en interpretación de conferencia, sí incluyen un componente bastante sustancial en dicha materia. Tal es el caso del Máster en Traducción, Interpretación y Estudios Interculturales ofertado por la UAB, con una especialidad en Interpretación Profesional que, en la práctica, viene a ser interpretación de conferencia, con 20 créditos de consecutiva y 15 de simultánea.

\subsection{Otros títulos de posgrado con componentes de interpretación}

Hay una serie de universidades que ofertan títulos de máster más amplios, con algunas asignaturas de interpretación, aunque no es su objetivo proporcionar una formación especializada y profesional. Tal sería el caso de la UPO, que oferta un Máster en Comunicación Internacional, Traducción e Interpretación que incluye 6 créditos de Interpretación Consecutiva y Simultánea, o la UPF con su Máster en Estudios de Traducción, con una mención en Traducción Especializada e Interpretación. Esta titulación dedica 15 créditos a las asignaturas de interpretación, las cuales tienen denominaciones bastante generales. En la misma línea la UA oferta un Máster Universitario en Traducción Institucional con 5 créditos de interpretación por par de lenguas. ${ }^{6}$

Es interesante constatar la presencia de asignaturas optativas de interpretación en los servicios públicos en los nuevos títulos de máster. Al igual que en el Grado, demuestra la creciente concienciación en cuanto a este tipo de interpretación y la necesidad de una formación específica.

La iniciativa más conocida y pionera en formación en ISP es seguramente la de la Universidad de Alcalá (UAH), donde se han venido impartiendo cursos propios de posgrado en Traducción e Interpretación en los servicios públicos en una amplia gama de combinaciones lingüísticas desde hace muchos años. Ahora estos cursos propios coexisten con un Máster Universitario que dedica 13 de sus 60 créditos a la formación en interpretación para estos ámbitos.

El recurso de los títulos propios ha resultado ser muy eficaz en la formación en ISP, sin duda por la flexibilidad que ofrece. Esta fórmula se ha empleado en la UJI que, durante varios años seguidos, ofertó un título propio en Mediación Intercultural e Interpretación en Ámbitos Sanitarios, y también

6. En inglés estos créditos se dedican a la enseñanza de la interpretación judicial/policial/ jurada mientras que en las combinaciones con francés y alemán se centran en la interpretación en organizaciones internacionales. 
en la UAB, con su I Curso de Interpretación en los Servicios Públicos, que ahora ha pasado a formar parte de un título de posgrado en traducción jurídica. Igualmente, durante muchos años, la ULL ha ofertado un título de Experto Universitario en Traducción e Interpretación para los Servicios Comunitarios. Lamentablemente, estas últimas iniciativas se ven interrumpidas por falta de matrícula y no siempre está garantizada su impartición.

\subsection{Interpretación en ámbitos judiciales}

Hay un campo de la ISP que va a adquirir cada vez más importancia en los próximos años y es la Interpretación en ámbitos judiciales. La directiva 2010/64 de la UE relativa al derecho a traducción e interpretación en los procesos penales ya está en vigor y el plazo para que los países miembros realizaran la transposición a la legislación nacional cumplió en octubre de 2013. El objetivo de esta directiva es salvaguardar los derechos procesales mediante la adecuada formación y acreditación de los traductores e intérpretes.

La transposición de esta Directiva a la legislación española requiere la elaboración de un sistema para la realización de dicha acreditación y formación. ${ }^{7}$

De momento, hay pocas universidades que dedican algunos créditos a la formación de intérpretes judiciales en sus programas de posgrado, entre ellas la UA, la UAH, la UAB, la ULL y la UPCO. Esta escasa atención prestada por las universidades españolas a la interpretación judicial no es de extrañar teniendo en cuenta la lamentable situación de las contrataciones para la provisión de este servicio en España en la actualidad. Sin embargo, a raíz de la Directiva, es de prever que esta rama se convertirá en un campo más profesionalizado en un futuro no demasiado lejano y que esto requerirá nuevas iniciativas formativas desde las universidades (Ortega Herráez 2011).

\section{Análisis}

Hemos procurado ofrecer una panorámica de la formación en interpretación en España desde sus inicios en la década de los 70 del siglo pasado hasta la fecha actual.

En lo que respecta a las Directrices Generales Comunes del plan de estudios de la Licenciatura (troncalidad), en teoría al menos, encerraban una contradicción al incluir una formación obligatoria en interpretación consecutiva

7. Parte de la transposición se realizará aprovechando el Proyecto de Ley de Reforma de la Ley de Enjuiciamiento Criminal. Previsiblemente, la normativa que rige el Registro de Traductores e Intérpretes acreditados será objeto de una ley específica para este propósito. 
y simultánea de solo 180 horas para estudiantes que no necesariamente tuvieran la suficiente competencia lingüística y de conocimientos generales para ello y, en algunos casos, con carencias en cuanto al equipamiento técnico necesario y con grupos excesivamente numerosos.

Cabe preguntar si esta formación obligatoria estaba ajustada a las perspectivas profesionales y motivaciones de la mayoría de los licenciados. Los datos disponibles demuestran que las salidas laborales de los titulados en Traducción e Interpretación no se limitan de ninguna manera a la traducción e interpretación, sino que abarcan numerosos campos tales como entornos empresariales, comerciales, turísticos, banca o importación-exportación (Kelly 2005, Calvo Encinas, Kelly \& Vigier 2008).

Por otra parte, los datos demuestran que, hasta hace poco, ningún licenciado de las universidades españolas había conseguido aprobar los exámenes de interpretación de las instituciones europeas habiendo cursado solo las asignaturas troncales de Técnicas de Interpretación Consecutiva y Técnicas de Interpretación Simultánea (Ribot 2010). La gran mayoría de los licenciados de universidades españolas que han superado dichos exámenes lo han hecho después de hacer un máster posterior en Interpretación de Conferencia, con la salvedad de aquellos licenciados de la UGR que habían cursado el itinerario optativo de especialización en Interpretación de Conferencia con una duración de 400 horas que se ofertaba en dicha universidad.

En cuanto a las motivaciones, cabría pensar que no todos los estudiantes matriculados en la Licenciatura en Traducción e Interpretación buscaban una especialización concreta en esos campos, sino más bien una carrera relacionada con la vertiente práctica y actual de los idiomas modernos y los países donde se hablan dichos idiomas, como alternativa a los estudios filológicos, que eran la única opción para aquellos que querían estudiar lenguas en España.

Vistos estos datos se podría aventurar que la Licenciatura, por lo menos sobre el papel, perdió la oportunidad de ofrecer a los estudiantes una introducción a la comunicación oral interlingüística y la interpretación (en general, no la interpretación de conferencia), lo cual, en primer lugar, les hubiera ayudado a decidir si querían pasar a una posterior especialización o no y, en segundo lugar, les hubiera proporcionado una formación en interpretación para aquellas situaciones en las que previsiblemente podrían llegar a actuar como enlace lingüístico ocasional en su futura vida profesional en uno de los ámbitos mencionados arriba. La adquisición de la técnica de interpretación de enlace no solo hubiera resultado más asequible para los estudiantes, teniendo en cuenta el número limitado de horas y otros condicionantes que hemos 
mencionado, sino que hubiera resultado más realista y ajustada en cuanto a sus posibles perspectivas laborales y sus propias motivaciones.

$\mathrm{Al}$ margen de las cuestiones relacionadas con otros prerrequisitos para la formación en Interpretación (dominio de las competencias instrumentales, número de horas, tamaño de grupos, metodología docente), es dudoso que la formación en interpretación de conferencia, y sobre todo en simultánea, pueda ser eficaz si es obligatoria. Las exigencias son considerables durante esta formación y los estudiantes deben estar plenamente motivados para poder invertir la ingente cantidad de tiempo y energía necesarios si han de cumplir los objetivos pedagógicos, aún en las mejores condiciones.

En el mejor de los casos, (suponiendo que tanto la infraestructura disponible como el número de estudiantes por grupo y las competencias de partida de los mismos fueran óptimas), los 9 créditos de la asignatura troncal de la Licenciatura solo permitían ofrecer una introducción a la simultánea. Cabe preguntarse, pues, ¿de qué sirve una introducción a la simultánea sin la posibilidad de llegar más allá?; ¿qué objetivo formativo se cubre al ofertar "un aperitivo" de interpretación simultánea?; ¿cómo se objetivan las competencias necesarias?; ¿cómo se evalúa la adquisición de dichas competencias? La interpretación simultánea es una actividad cognitiva compleja que requiere una formación muy seria, rigurosa y relativamente larga en el tiempo, y unas condiciones de partida muy exigentes en cuanto al dominio lingüístico. No tratarlo como tal es un engaño para los estudiantes, ya que les induce a creer, erróneamente, que están preparados para el desempeño profesional, y les produce una gran frustración, lo cual precisamente puede tener un efecto perjudicial no solo para ellos personalmente sino para la reputación de las universidades españolas en general y la profesión en su conjunto.

En cuanto al Grado, a juzgar por los datos recabados en este estudio, no se percibe una clara apuesta por esa titulación generalista a la que se refieren los Descriptores de Dublín (VV.AA.). Ciertamente la mayor flexibilidad en cuanto al plan de estudios ha arrojado un panorama muy variado en el que la mayoría de las universidades parecen haberse alejado del modelo impuesto de la Licenciatura, aunque en algunos casos se han acercado más.

De hecho, si se tienen en cuenta las universidades que ofertan itinerarios optativos de especialización y las que ofertan créditos obligatorios de técnicas propias de la interpretación de conferencia, así como el limitado éxito de los títulos de máster, parecería que la mayoría de los centros implicados han decidido ofertar una formación en interpretación de conferencia dentro del Grado. Esto se hará con mayor o menor acierto ya que en muy pocos casos se acerca a las 400 horas de clases presenciales que recomienda el consorcio 
europeo. Esto no supone un problema en sí y muy probablemente es una lógica respuesta a las dificultades y al coste asociados a las titulaciones de máster. El problema en el pasado -y que quizás convendría evitar en el presente y en el futuro- ha sido ofertar esta especialidad de forma obligatoria y poco realista, sin los prerrequisitos necesarios, como si se planteara enseñar a los futuros cirujanos a operar antes de haber estudiado anatomía (Mayoral Asensio 1998, Kelly 1996).

\section{Conclusiones}

A modo de conclusión, podemos afirmar que la formación en Interpretación ofertada en las universidades españolas en la actualidad difiere mucho de un centro a otro. Sigue centrándose en el Grado y el número de créditos obligatorios en Interpretación oscila entre 6 y 30, dependiendo de la universidad. Por un lado, hay universidades que ofrecen un mínimo de créditos obligatorios, a modo de introducción, y la posibilidad de especializarse posteriormente en itinerarios optativos más o menos especializados. Por otro lado, hay universidades que reproducen el modelo de la Licenciatura e incluso ahondan en él, incluyendo una cantidad significativa de créditos obligatorios en interpretación. Es cierto que solo hay siete universidades que incluyen el mismo número de créditos obligatorios o más que la Licenciatura, pero al mismo tiempo en casi la mitad de las universidades estudiadas sigue habiendo créditos obligatorios en interpretación simultánea sin que, por otra parte, y según los criterios ya explicados, sean suficientes para poder formar profesionales a un nivel adecuado. Si bien muchas universidades han elegido nombres generales para las asignaturas obligatorias de Interpretación, que aluden al carácter introductorio de la asignatura, en nueve centros los nombres de las asignaturas obligatorias siguen refiriéndose a la interpretación consecutiva o simultánea.

En cierta manera, este panorama dispar refleja la poca claridad conceptual con la que nació el proyecto de Grado en cuanto a la interpretación. Frente a las posturas que proponían la erradicación de la interpretación del nuevo Grado, la comisión encargada de valorar la titulación optó por mantenerla, y no solo la interpretación de enlace. Sin embargo, basa esta recomendación en un razonamiento ciertamente curioso y a nuestro juicio, contradictorio:

Puesto que la intención es, siguiendo las directrices de Bolonia, proponer un título generalista, la mesa opina que debería mantenerse la interpretación (no solo de enlace) de manera introductoria en el grado (Aneca 2004: 8).

Siendo la interpretación profesional una especialización en sí es difícil entender dicho razonamiento, sobre todo teniendo en cuenta que la técnica de 
interpretación de enlace se concibe precisamente como introducción. La confusión resultante se refleja en los actuales planes de estudios.

Al parecer, la mayoría de las universidades que han intentado ofertar una formación en interpretación de conferencia a nivel de posgrado están encontrando obstáculos que han impedido la puesta en marcha o la continuidad de dichas titulaciones, por lo que esta opción no se ha consolidado por el momento. Es evidente que la educación superior no está precisamente en su mejor momento y que la situación económica adversa que atraviesa España, con los drásticos recortes y la consiguiente falta de recursos entre la población, deja sus efectos. Si bien es cierto que los títulos oficiales son menos costosos que los propios, esta diferencia parece relativa en tiempos de grave crisis económica. Además, la solicitud y verificación de los títulos es un proceso complejo y burocrático, que resulta poco flexible y requiere una inversión ingente de tiempo por parte del equipo que promueve la titulación, condicionantes que no invitan a seguir esta modalidad. En todo caso, estamos en una fase inicial de la implantación del EEES, por lo que es previsible que la situación pueda evolucionar.

Una de las novedades más interesantes del Grado es la inclusión de asignaturas optativas en otros géneros de interpretación aparte de la interpretación de conferencia, sobre todo la interpretación en los servicios públicos. Sin embargo, la formación especializada en interpretación judicial, que va a ser necesaria en los próximos años, no está cubierta de momento.

Queda por ver cuál será el desarrollo en la práctica de los grados que ahora mismo inician su andadura. Sería deseable que se pudiera ofertar una formación en todos los géneros de interpretación, de una manera equitativa, haciendo un uso racional de los recursos, posibilitando a todo el que lo desee la oportunidad para adquirir las competencias previas necesarias para emprender esa formación. Lo ideal sería que la formación en sí, sea en grado o en posgrado, se imparta teniendo en cuenta las pautas que la experiencia, tanto la nuestra como la de otros países, nos ha legado, para así cumplir lo mejor posible con los objetivos que nos marcamos.

\section{Referencias bibliográficas}

ANECA. (2004) Libro Blanco. Título de Grado en Traducción e Interpretación. Versión electrónica: <http://www.aneca.es/var/media/150288/libroblanco_ traduc_def.pdf>

Association Internationale des Interprètes de Conférence (AIIC). Versión electrónica: <www.aiic.org> 
Calvo Encinas, Elisa. (2009) Análisis curricular de los estudios de Traducción e Interpretación en España. Tesis doctoral. Universidad de Granada.

Calvo EnCINAS, Elisa; Dorothy Kelly \& Francisco Vigier. (2008) "Diseño curricular en TI: reflexiones a la luz de los datos de inserción laboral." En: Navarro, Fernando et al. (eds.) La traducción: balance del pasado y retos del futuro. Alicante: Aguaclara.

European Master's in Conference Interpreting (EMCI). Versión electrónica: $<$ www.emcinterpreting.org>

FABBRO, Franco; Laura Gran; Gianpaolo Basso \& Antonio Bava. (1990) "Cerebral Lateralization in Simultaneous Interpreting." Brain and Language 39:1, pp. 69-89.

FABBRO, Franco \& Laura Gran. (1994) "Neurological and neuropsychological aspects of polyglossia and simultaneous interpreting." En: Lambert, Sylvie \& Barbara Moser-Mercer (eds.) 1994. Bridging the Gap. Empirical Research in Simultaneous Interpretation. Amsterdam \& Filadelfia: John Benjamins, pp. 273-318.

GILE, Daniel. (1985) "Le modèle d'efforts et l'équilibre d'interprétation en interprétation simultanée." Meta. Numéro Spécial: Interprétation de Conférence 30:1, pp. 44-48.

GILE, Daniel. (1991) "The Processing Capacity Issue in Conference Interpretation." Babel 37:1, pp. 15-27.

GILE, Daniel. (1995) Basic Concepts and Models for Interpreter and Translator Training. Amsterdam \& Filadelfia: John Benjamins.

Gillies, Andrew. (2004) Conference Interpreting - A New Students' Companion. Cracovia: Tertium.

Gillies, Andrew. (2013) Conference Interpreting - A Students' Practice Book. Londres \& Nueva York: Routledge.

GrAN, Laura. (1989) "Interdisciplinary Research on Cerebral Asymmetries: Significance and Prospects for the Teaching of Interpretation." En: Gran, Laura \& John Dodds (eds.) 1989. The Theoretical and Practical Aspects of Teaching Conference Interpretation. Udine: Campanotto Editore, pp. 93-100.

Grupo Comunica. Observatorio Permanente sobre Traducción e Interpretación en los Servicios Públicos. Versión electrónica: <http://red-comunica.blogspot. com>

KELly, Dorothy. (1996) "El plan de estudios de la Licenciatura en Traducción e Interpretación de la Universidad de Granada: elaboración, limitaciones y perspectivas de futuro." En: Lozano, Wenceslao et al. (eds.) 1996. Actas de las primeras jornadas sobre diseño curricular del traductor e intérprete. Granada: Universidad de Granada, pp. 9-29.

Kelly, Dorothy. (2005) A Handbook for Translator Trainers. Manchester: St Jerome. 
KelLY, Dorothy y Anne Martin. (2009) "Training and Education." En: Baker, Mona \& Gabriela Saldanha (eds.) 2009. Routledge Encylopedia of Translation Studies $2^{\text {nd }}$ Edition. Londres \& Nueva York: Routledge.

LAMBERT, Sylvie. (1989) "Recall and Recognition among Conference Interpreters." En: Gran, Laura \& John Dodds (eds.) 1989. The Theoretical and Practical Aspects of Teaching Conference Interpretation. Udine: Campanotto Editore, pp. 83-92.

MARTin, Anne. (2003) "La direccionalidad y la interpretación: epílogo." En: Kelly, Dorothy et al. (eds.) 2003. La Direccionalidad en Traducción e Interpretación. Perspectivas teóricas, profesionales y didácticas. Granada: Atrio.

MaYoral Asensio, Roberto. (2007) "For a New Approach to Translator Training. Questioning Some of the Concepts which Inform Current Programme Structure and Content in Spain." The Translator and Interpreter Trainer 1:1, pp. 79-95.

MinisTeRio deEdUCACIÓN Y CienCia. (1991) Real Decreto 1385/1991, de 30 deagosto, por el que se establece el título universitario oficial de Licenciado en Traducción e Interpretación. Boletin Oficial del Estado, pp. 31773-31775. Versión electrónica: <http://www.boe.es/buscar/doc.php?id=BOE-A-1991-24111>

Nolan, James. (2005) Interpretation: Techniques and Exercises. Clevedon: Multilingual Matters.

Ortega Herráez, Juan Miguel; Ma Isabel Abril Martí \& Anne Martin. (2009) "Community Interpreting in Spain." En: Hale, Sandra Beatriz; Uldis Ozolins \& Ludmilla Stern (eds.) 2009. The Critical Link 5. Quality in Interpreting - A Shared Responsibility. Amsterdam \& Filadelfia: John Benjamins, pp. 149-167.

Ortega HerRÁEZ, Juan Miguel. (2011) "Cómo acreditar intérpretes a través de la traducción: análisis crítico de la acreditación de intérpretes jurídicos en España." TRANS. Revista de traductología 15, pp.131-153. Versión electrónica: <http://www.trans.uma.es/pdf/Trans_15/131-153.pdf>

Padilla Benítez, Presentación. (1996) Procesos de memoria y atención en la interpretación de lenguas. Tesis doctoral. Universidad de Granada. (microforma).

PÖCHHACKER, Franz. (1995) "Simultaneous Interpreting: A Functionalist Perspective." Hermes Journal of Linguistics 14, pp. 31-53.

Riвot, Alfonso. (2010) "La interpretación en las instituciones europeas." Conferencia impartida en la Facultad de Traducción e Interpretación, Universidad de Granada, marzo 2010.

RITAP (RED DE TRADUCTORES E INTÉRPRETES DE LA ADMINISTRACIÓN PÚBLICA) Y APTIJ (ASOCIACIÓN PROFESIONAL DE TRADUCTORES E INTÉRPRETES JUDICIALES Y JURADOS). (2011) Libro blanco de la traducción y la interpretación institucional. Madrid: Ministerio de Asuntos Exteriores y de Cooperación. Versión electrónica: <http://www.ritap.es/recomendaciones-y-libro-blanco/> 
SeleskovitCH, Danica. (1968) Les Interprètes dans les Conférences Internationales. París: Lettres Modernes Minard.

Seleskovitch, Danica. (1975) Langage, Langues et Mémoire. París: Lettres Modernes Minard.

SEleskovitCH, Danica. (1981) "L'enseignement de l'interprétation." En: Delisle, Jean (ed.) 1981. L'enseignement de l'interprétation et de la traduction: de la théorie à la pédagogie. Ottawa: Éditions de l'Université d'Ottawa, pp. 23-46.

SEleskovitch, Danica. (1985) "Interprétation ou interprétariat?" Meta. Numéro Spécial: Interprétation de Conférence 30:1, pp. 19-24.

Seleskovitch, Danica \& Marianne Lederer. $(1989,2002)$ Pédagogie Raisonnée de l'Interprétation. Bruselas \& Luxemburgo: Didier Érudition.

VV.AA. Descriptores de Dublín. Versión electrónica: <http://www.thematicnetworkdietetics.eu/everyone/1926/5/0/30>

\section{NOTA BIOGRÁFICA / BIONOTE}

ANNE MARTIN es Licenciada en Traducción e Interpretación por la Universidad de Heriot-Watt (Edimburgo) y Doctora en Traducción e Interpretación por la Universidad de Granada. Es Profesora Titular de Universidad de la UGR y coordinadora del Máster en Interpretación de Conferencias de la misma universidad. Es miembro del grupo de investigación GRETI (La interpretación ante los retos de la mundialización: formación y profesión), financiado por la Junta de Andalucía, así como miembro del Grupo Comunica, Observatorio Permanente sobre Traducción e Interpretación en los Servicios Públicos. Fue co-editora de la revista Puentes. Hacia Nuevas Investigaciones en la Mediación Intercultural desde 2002 hasta 2010. Es intérprete de conferencia y miembro de AICE (Asociación de Intérpretes de Conferencia de España).

ANNE MARTIN has a B.A. (Hons) Languages (Translation and Interpreting) from Heriot-Watt University, Edinburgh, U.K., and a PhD in Translation and Interpreting awarded by the University of Granada, Spain. She is Senior Lecturer at the University of Granada and coordinator of the Master's Degree in Conference Interpreting at that same university. She is a member of the GRETI Research Group (Interpreting and the Challenges of Globalisation: Training and Professional Practice), funded by the Andalusian Regional Government and also a member of the Comunica Network (Permanent Observatory on Community Translation and Interpreting). Between 2002 and 2010 she was co-editor of Puentes. Hacia Nuevas Investigaciones en la Mediación Intercultural Conference Interpreter. She is a member of AICE (Spanish Professional Association of Conference Interpreters). 